INVESTIGACIÓN/RESEARCH

\title{
REDES SOCIALES Y DOCENCIA EN LOS ESTUDIOS DE PUBLICIDAD
}

Emma Torres Romay: Universidad de Vigo. España emmatr@uvigo.es

Juan Manuel Corbacho Valencia2: Universidad de Vigo. España jmcorbacho@uvigo.es

\section{RESUMEN}

El fuerte incremento de la penetración de internet en España supone también un gran reto para el contexto educativo. En este trabajo intentamos realizar una reflexión sobre la integración de las redes sociales en la formación universitaria, pero desde una perspectiva muy particular: la derivada del hecho de que las redes sociales pueden contribuir a un proceso formativo pero también, en el caso de la titulación en Publicidad y Relaciones Públicas, como una herramienta más del futuro profesional de los egresados. La finalidad es aportar conclusiones operativas sobre ambas cuestiones $\mathrm{y}$, para ese fin, se ha examinado profundamente la bibliografía y los estudios existentes sobre la utilización de las redes sociales en la docencia y, posteriormente, se aportan los parámetros básicos sobre el modelo de aplicación de las redes sociales en la actividades docentes, un modelo que sigue las líneas preexistentes pero que, además, añade nuevas perspectivas o elementos de valoración. De esta forma, la experiencia relatada se centra en una perspectiva muy concreta - las redes sociales - de la evolución de los métodos docentes en la universidad y, por tanto, deja fuera muchas otros elementos relativos a las nuevas tecnologías (wikis, blogs, webs...) que probablemente necesiten un estudio específico.

PALABRAS ClAVE: Redes sociales - Docencia - Publicidad - Nuevas tecnologías Metodologías

\footnotetext{
1 Autor correspondiente:

Emma Torres Romay: Facultad de Ciencias Sociales y de la Comunicación. Universidad de Vigo. Pontevedra. España.

Correo: emmatr@uvigo.es

${ }^{2}$ Juan Manuel Corbacho Valencia: Facultad de Ciencias Sociales y de la Comunicación. Universidad de Vigo. Pontevedra. España.

Correo: jmcorbacho@uvigo.es
} 


\title{
SOCIAL NETWORKS FOR LEARNING IN ADVERTISING STUDIES
}

\begin{abstract}
The sharp increase in internet penetration in Spain is also a major challenge for the educational context. In this paper we attempt to make a reflection on the integration of social networks in university education, but from a very particular perspective: the derivative of the fact that social networks can contribute to a learning process but also, in the case of the titration Advertising and Public Relations, as a tool of the future career of the graduates. The purpose is to provide operational conclusions on both issues and, to this end, the literature has examined deeply and existing studies on the use of social media in teaching and subsequently provide the basic parameters of the model application social networks in teaching, a model that follows the existing lines but also adds new perspectives or elements of assessment. Thus, the related experience focuses on a very specific perspective - social networks - the evolution of teaching methods in college and therefore leaves out many other elements relating to new technologies (wikis, blogs, websites ...) that are likely to require a specific study.
\end{abstract}

KEY WORDS: Social Networking - Teaching - Advertising - New technologies Methodologies

\section{INTRODUCCIÓN}

En España el $12 \%$ de los hogares tiene acceso a internet ${ }^{3}$, cifra muy inferior a la Europea pero que supone que la red tiene actualmente un índice de penetración (según las fuentes más optimistas) del 70\%, aproximadamente unos 29 millones de ciudadanos ${ }^{4}$. Lo más llamativo de estos datos está en el rápido crecimiento de este medio, que ha subido a un ritmo de más de $10 \%$ de penetración año tras año.

Todas las previsiones indican que falta muy poco para que la red se convierta en el segundo medio, sólo por detrás de la televisión (según datos de Infoadex). Por tanto, es en un momento especialmente complejo en lo referido a la gestión de esta nueva vía de comunicación con unas grandes potencialidades tanto de carácter social como, por supuesto, educativas.

Esta preocupación se ha generalizado en todo el mundo, el Reino Unido ya ha anunciado que reformará la educación primaria para adaptarse a las denominadas

\footnotetext{
3 "El índice de penetración de internet en España es inferior al de la Unión Europea" en Puro Marketing. 13 de octubre de 2010. Disponible en: http://www.puromarketing.com/18/8176/indice-penetracioninternet-espa-inferior-union.html [27-02-2011]

4 "Informe de internet en España y en el Mundo. Situación actual Redes Sociales en España" en Consultora Tatum. Mayo de 2010.
} 
nuevas tecnologías ${ }^{5}$. Sin embargo, la Universidad parece estar respondiendo con más lentitud que la educación básica quizá debido a la propia naturaleza de la institución, en la que los avances son abordados desde la base. Se habla de Nuevas Tecnologías y educación pero no de nuevas tecnologías en la Universidad ${ }^{6}$.

La mayor parte de las iniciativas en este sentido guardan relación con la implementación de las denominadas plataformas de teledocencia (Marín y Maldonado, 2010), herramientas que son diseñadas específicamente por cada una de las universidades en base a una serie de modelos y sobre las que, hasta ahora, tampoco parecen haberse realizado análisis generales de importancia.

En este contexto, debemos, de nuevo, tener en cuenta la realidad social de las nuevas tecnologías. Dentro de todos los servicios posibles que ofrece internet las redes sociales han cobrado un especial protagonismo. Del total de usuarios de internet en España el $80 \%$ pertenece a alguna red social, según el AIMC, y de todas las personas presentes en esas redes, el 89\% tiene perfil en Facebook, el 60\% en Youtube, un 41\% en Tuenti y el 19\% en Twitter, según datos de 2010 del IAB.

Y, como no podía ser de otra manera, a la cabeza de la revolución mediática están las personas más jóvenes. Las redes sociales tienen su mayor mercado entre las personas de entre 10 a 18 años, según se concluye del estudio "Menores y Redes Sociales" elaborado por el Foro de Generaciones Interactivas 7 . La IAB indica que los chicos y chicas de entre 18 y 24 años acceden a estas redes todos los días (90,8\%) y, probablemente, le dediquen mucho más tiempo que los métodos de formación convencionales.

De esta forma, podemos situar aquí uno de los grandes retos para la educación en el futuro: el saber establecer un uso correcto de las nuevas tecnologías pero, más concretamente, de las redes sociales (Jimenez, 2011), de manera que beneficie el proceso formativo también en el nivel universitario. Sin embargo, nuestro objeto de estudio tiene ciertos matices con respecto a este punto de partida.

\section{METODOLOGÍA}

En este artículo pretendemos abordar dos problemáticas paralelas, por una parte el aprovechamiento de las redes sociales en el proceso formativo a nivel universitario y, por otra, el desarrollo de fórmulas para su empleo en campañas de comunicación y de publicidad.

El porqué de este doble objeto de estudio guarda relación directa con el contexto en el

\footnotetext{
5 “El Reino Unido reformará la educación primaria para adecuarla a las Nuevas Tecnologías” en El País. 25 de marzo de 2009.

${ }^{6}$ Los estudios más profundos sobre esta relación datan de 2003 (Cabero)

7 "El 35\% de los menores internautas tienen dos o más perfiles en las redes sociales" en Educaweb.com. 28 de enero de 2011. Disponible en: http://www.educaweb.com/noticia/2011/01/28/35-menoresinternautas-tienen-dos-mas-perfiles-redes-sociales-14579.html 27-02-2011
} 
que se ha puesto en marcha el proyecto, el Grado en Publicidad y Relaciones Públicas de la Universidad de Vigo. Los egresados de este título deberán conocer profundamente estas redes sociales como nuevo soporte publicitario e internet como uno de los fundamentales medios de comunicación en la creación de estrategias y, por lo tanto, el empleo de las mismas va más allá de los conocimientos que deberían tener los estudiantes de otro tipo de titulaciones.

Desde este punto de vista, la puesta en marcha de un proyecto docente en que se tenga en cuenta los dos objetivos planteados supone partir de una serie de hipótesis iniciales:

- Los estudiantes conocen perfectamente el medio y el soporte, pero parten de un empleo frívolo y poco relevante del mismo. De hecho, casi todos los informes hablan de que el uso preferente de las redes está en las actividades de carácter lúdico y social (por ejemplo en el de IAB y Elogia).

- El empleo de las redes sociales en la docencia supone un acercamiento excesivamente personal a los estudiantes, que parten de un concepto de intimidad mucho más abierto que en generaciones anteriores (Serraller, 2009). Según datos de INTECO, el 43\% de los usuarios no ponen límites a sus contenidos en la red.

- Las principales aportaciones existentes en torno al uso de internet en la docencia parten de la aplicación de modelos clásicos que se adaptan a esta nueva herramienta pero que, en muchos casos, no tienen en cuenta las diferencias comunicativas e incluso conceptuales que estas suponen. De esta forma, parecen estarse difundiendo los mismos contenidos pero por otras vías diferentes.

- Son pocos los alumnos o alumnas que se acercan a las redes sociales como una herramienta de trabajo $\mathrm{y}$, aquellos que lo hacen, demuestran tener un pobre conocimiento de la realidad "comercial" de este soporte. Así, nuevas figuras profesionales, como la de community manager, son entendidas de forma inadecuada (Castellón, 2011).

A partir de estas ideas iniciales, la metodología propuesta pasa por diversas fases. En una primera se realizará un seguimiento de las propuestas preexistentes (estado de la cuestión) para, a continuación, poner en marcha una experiencia concreta de uso de redes sociales en el caso concreto de la materia Estrategias de la Comunicación Publicitaria y, por último, algunas aportaciones en torno al empleo de las redes en el desarrollo del proyecto final de esta materia.

\section{ANÁLISIS Y DISCUSIÓN}

\subsection{Apuntes básicos sobre el empleo de las redes sociales en la docencia}

Si realizamos una búsqueda intensa de bibliografía, hemerografía o experiencias sobre el empleo de las redes sociales en la docencia debemos incidir en la consideración, ya realizada, en torno al hecho de que la mayor parte de la documentación disponible está centrada en las consecuencias posibles (De Pablos y Villanueva, 2005) de la implementación de las nuevas tecnologías de la información e la comunicación en 
docencia (Salinas, 2004) o en el uso de las plataformas de teledocencia creadas específicamente por cada universidad.

En lo referido a estas plataformas en la mayoría de los casos se han gestionado como distribuidoras de documentación y forma de contacto de los estudiantes (Bautista, Borges y Forés, 2004). En lo referido a la Universidad de Vigo, desde el año 2003 se emplea el recurso denominado Faitic, soportado en la plataforma Claroline, pero se trata de formatos o estructuras muy alejados del concepto de red social.

Si nos centramos concretamente en lo que se refiere a las redes sociales tenemos que destacar que, como es habitual, la tecnología va delante del avance real de su aplicabilidad. De hecho, encontramos referencias académicas sobre webblogs u otras herramientas $^{8}$, incluidas las webs como formato genérico. En este sentido, no se cuenta aún con trabajos sobre las redes sociales aplicadas a la docencia universitaria pero si contamos con las primeras aproximaciones para lo relativo a metodologías para la enseñanza de publicidad.

Este hecho constata nuestro planteamiento inicial respecto a que en estos estudios la necesidad de formación en este ámbito es, si cabe, doblemente importante. De hecho, los estudios publicados indican toda una serie de ventajas claras de las redes sociales frente a otras herramientas de la web 2.0 y sus evoluciones posteriores (Meso Ayerdi y otros, 2011) incidiendo en la adecuación de su uso para los estudios de publicidad y relaciones públicas.

\subsection{La experiencia de las redes sociales en los estudios de publicidad}

Una vez determinada la importancia de las redes sociales en el ámbito educativo - al tiempo que se constata el lento avance de los modelos de aplicación - es necesario describir la experiencia:

\subsubsection{Planteamiento de la propuesta}

La propuesta se inició en el curso 2010-2011 para la materia de segundo curso del Grado Estrategias de la Comunicación publicitaria aplicando los siguientes pasos:

- Investigación previa del "mercado". Se recabaron datos sobre la presencia de los alumnos y alumnas en las redes sociales. La totalidad de los estudiantes de las materia declararon ser usuarios de las redes sociales (52 personas) de ese total un 92\% empleaban Facebook, un 65\% empleaban dos (Facebook y Tuenti) y un pequeño porcentaje - 18\% - son usuarios de Twitter. Estas cifras están por encima de los datos que indican que el $41 \%$ de los españoles entre 18 y 24 años son usuarios de redes sociales $(\mathrm{IAB}, 2009)$ pero da una referencia del necesario carácter vanguardista de los universitarios.

\footnotetext{
${ }^{8}$ Número monográfico (2005) "XII Jornadas Universitarias de Tecnología Educativa. Universidad de Cádiz" en Revista Latinoamericana de Tecnología Educativa. Vol. 4. №1.
} 
- Investigación previa de la "competencia". También se tuvo en cuenta el hecho de si otros profesores empleaban este recurso en su actividad. Del total de docentes en el momento de iniciar el proyecto, 115, sólo uno incluía en su ficha de contacto referencias a redes sociales, mientras que eran 15 los que hacían referencia a una página web personal y uno a un blog9. De manera más informal, fuera de las fichas de los docentes, queda constancia de que la mayor parte de ellos son usuarios de redes sociales pero sólo se confirma un caso de un profesor que la emplee con finalidades docentes de carácter no formal.

- Elaboración del "producto". Una vez que sabemos que la fórmula era válida y que la plataforma más adecuada era Facebook, se procedió a la configuración del espacio que debería albergar la iniciativa, teniendo siempre muy presentes ejemplos y experiencia previas (Piscitelli y otros, 2010). En este sentido, el problema estaba en discernir si resulta más correcta la creación de un perfil o de una página. Resulta una decisión compleja que finalmente se concretó en un perfil debido a que este permite mayor interacción con los miembros que participan en él (Serrano, 2008).

- Captación del "público objetivo". Tras la puesta en marcha de la iniciativa10, era necesario conseguir que los estudiantes participaran en ella. Después de la correspondiente comunicación de la existencia del espacio en el aula, se inició un proceso de búsqueda de estudiantes. Sólo fue necesario remitir la invitación a cinco de los matriculados para que, inmediatamente se consiguieran los enlaces de hasta 25 personas más del aula. Bien es cierto que era necesario buscar contenidos iniciales de "enganche" para que se encontraran elementos de referencia e interés en el perfil. De esta forma, la primera iniciativa estuvo en colgar fotografías de los trabajos finales de cursos anteriores ${ }^{11}$.

- Organización de los objetivos del proyecto. De formar paralela a la captación de los estudiantes, era necesario contar con una definición clara de los objetivos perseguidos con esta herramienta. Para este fin se estableció un debate con los alumnos y alumnas que, entre otros elementos, subrayaron que esta vía era mucho más efectiva para el envío de avisos que el habitual correo electrónico ya que la forma de conexión a esta red es permanente y en tiempo real12. Además de estas cuestiones más operativas, también se constató el interés de que esta red sirviera, especialmente, para trabajar dos de las competencias básicas de la materia ("Reconocer los elementos que componen el proceso publicitario e identificar sus funciones concretas"; “Adquirir conocimientos sobre todos los procesos posibles desarrollados en una agencia de publicidad"13).

- Establecimiento de fórmulas de control. Finalmente, para que la experiencia puesta

\footnotetext{
${ }^{9}$ Datos obtenidos a partir de la web del centro: http://www.csc.uvigo.es

10 Perfil de Facebook: Estratexias da Comunicación.

${ }^{11}$ Uno de los trabajos finales de la materia consiste en la elaboración de un "diccionario" de términos publicitarios específicos que los estudiantes elaboran en grupo y que deben presentar de una forma creativa y original, tal y como se puede ver en las imágenes colgadas en el perfil de Facebook antes referido. Cada curso se presenta alrededor de diez trabajos.

12 Recordamos que, según datos del IAB, el 90,9\% de los usuarios de redes sociales entre 18 y 24 años emplean las redes sociales diariamente.

${ }^{13}$ Según consta en la Memoria del Título de Grado en Publicidad y Relaciones Públicas, disponible en la web de la Universidad de Vigo:

http://webs.uvigo.es/vicprof/images/documentos/MEMORIAS_DEFINITIVAS/UVIGrado_Publicid ad_y_relaciones_publicas.pdf [27-02-2011]
} 
en marcha pueda tener cierta validez y proyección, era necesario establecer elementos de control y seguimiento que permitan identificar y corregir problemas. En este sentido, se establece una retroalimentación con el alumnado durante las sesiones de seminario y se emplearon las herramientas que la propia red Facebook facilita (estadísticas semanales e informes de actividad).

\subsubsection{Resultados iniciales de la propuesta}

Después de la puesta en marcha de la iniciativa, debemos valorar alguno de los resultados iniciales de la misma ya que, como indicamos, se trata de una iniciativa experimental e intuitiva que debe ser evaluada en profundidad para hacer las correcciones que sean necesarias. En este sentido tenemos que recoger las siguientes consideraciones:

- Inadecuado formato del producto. Como se ha indicado, en un primer momento se optó por el formato de perfil en lugar de página por sus mayores posibilidades de interrelación. Sin embargo, una vez puesta en marcha la experiencia, se debe tener en cuenta que el acceso al perfil depende de que los estudiantes acepten la invitación de amistad y esto supone que el docente-administrador del perfil puede acceder, a su vez, los perfiles de los estudiantes y, por tanto, la información personal. Se trata de un problema de carácter casi ético ya que complica el establecimiento de los límites necesarios entre profesor y alumno (Pozo y otros, 2006). Resulta muy llamativo que la mayor parte de los estudiantes cuenta con un perfil "abierto", es decir, que se puede acceder a su información y contenidos sin tener que solicitar su aceptación, dato que nos da una referencia del tipo de concepto que tienen de estas redes.

- Gran aceptación por parte del alumnado. Los estudiantes aceptaron ampliamente la propuesta. En sólo dos semanas el perfil contaba con 60 amigos, siendo notable la incorporación de antiguos alumnos y alumnas que enseguida se sumaron a la iniciativa. Esto supuso que se gestionaran grupos específicos, diferenciando entre los matriculados actualmente en la materia y los antiguos alumnos, añadiéndose un tercer grupo de otros docentes que solicitaron acceso al perfil.

- Divergencias en la interpretación de objetivos. En este punto es donde se encuentra la distorsión fundamental de la iniciativa. La mayor parte de los estudiantes ven en las redes sociales un elemento lúdico $\mathrm{y}$, como su propio nombre indica, de relación social ${ }^{14}$. Desde este punto de vista, y sumado a que todos ellos acceden con su perfil "personal" y no como un profesional o académico, supone que la mayor parte del tráfico generado sea de post informales (Corbacho, 2010).

- Mayor dinamismo y rapidez. La utilización del perfil de Facebook creado permite un mayor dinamismo en la gestión de contenidos docentes. Teniendo en cuenta que la materia Estrategias de la Comunicación Publicitaria se caracteriza por su constante evolución y por la necesidad de una renovación continúa; la red social permite actualizar los contenidos de una forma más directa y rápida. El sistema elegido para este caso fue el de hacer una selección semanal de informaciones recogidas de

14 "Los jóvenes y las redes sociales" en Marketing Directo. 19 de octubre de 2010.

http://www.marketingdirecto.com/actualidad/social-media-marketing/los-jovenes-y-las-redessociales/[18-08-2011] 
publicaciones especializadas, incluyendo links y referencias de estudios específicos. Tenemos que incidir, de nuevo, que Facebook se ha convertido en el sistema mucho más rápido para comunicarse con los alumnos y alumnas, haciendo que los avisos lleguen antes de lo que lo harían a través del correo electrónico.

- Poco seguimiento. A pesar del dinamismo y la rapidez antes referenciada, el problema está en que los estudiantes sí reciben la información transmitida a través de la red social (así queda demostrado en la utilización de estas referencias en las actividades prácticas y de seminario del aula) pero no generan flujo de conversaciones en el muro del perfil. De un total de 60 post realizados en los dos primeros meses del perfil, sólo se recibieron comentarios en tres casos; uno de esos comentarios fue realizado por otro docente; otro fue referido a un link con la entrevista de una antigua alumna actualmente presentadora de televisión y otro referido a una práctica de aula. En el muro sólo escribieron cuatro alumnos: uno comentando el resultado de una práctica, otros dos colgando vídeos de campañas publicitarias llamativas y el cuarto como resultado de un de los juegos que se usan en esa red. En resumen, el enlace de más éxito hasta ahora fue el que llevaba a una "entrevista profesional" creada para promocionar la serie televisiva Mad Men, ambientada en el mundo de la publicidad estadounidense de los años cincuenta ${ }^{15}$.

\subsection{Las redes sociales como contenido docente}

Después de todo esto, y teniendo en cuenta los problemas y potencialidades encontradas, no podemos pasar por alto el hecho de que los estudiantes con los que estamos trabajando en este proyecto son potenciales profesionales de la gestión de las redes sociales. La actual evolución del panorama publicitario (Infoadex) apunta claramente a que el futuro del sector pasa por su correcta adaptación a los denominados nuevos medios y, en especial, a las redes sociales ${ }^{16}$.

Se trata de un momento especialmente complejo desde la perspectiva formativa y profesional ya que no existen directrices claras sobre cómo va a evolucionar este mercado y resulta muy complejo adaptar contenidos tan variables e inestables a un programa docente concreto. Los propios docentes también son conscientes de esta realidad y muestran su inseguridad con respecto a las posibilidades laborales que le pueden ofrecer las redes sociales.

Como experiencia, durante el curso pasado se propuso una práctica (juego de rol) que consistía en la preparación de un curriculum profesional para presentar a una supuesta entrevista de trabajo. Los estudiantes debían elegir si presentar su curriculum para una plaza de director de arte, para una de ejecutivo de cuentas, para formar parte de un equipo de relaciones públicas o para puesto de community manager.

\footnotetext{
${ }^{15}$ En: http://www.amctv.com/originals/madmen/job-interview/[23-08-2011]

16 "La publicidad en las redes sociales es 40 veces más efectiva que la tradicional" en Marketing Directo. 17 de agosto de 2010. Disponible en:

http://www.marketingdirecto.com/actualidad/social-media-marketing/la-publicidad-en-redessociales-es-40-veces-mas-efectiva-que-la-tradicional/[23-08-2011]
} 
La realidad fue que muchos estudiantes manifestaron no tener demasiado claras las funciones de este tipo de profesionales. En este punto podemos afirmar, por tanto, que el claro desconocimiento del funcionamiento y evolución futura de los nuevos medios implica que el tratamiento de este asunto como contenido docente resulta difícilmente abordable en una programación de una materia. Por esta razón, la experiencia aquí expuesta, si bien ha registrado ciertos errores, resulta especialmente importante en la formación de futuros publicitarios.

\section{CONCLUSIONES}

La breve experiencia que aquí relatamos se centra en una perspectiva muy concreta de la evolución de los métodos docentes en la universidad y, por tanto, deja fuera muchas otras perspectivas sobre las nuevas tecnologías (wikis, blogs, webs...) que probablemente precisen un estudio específico. Las posibilidades de las redes sociales son claras en el ámbito de la docencia, pero ya desde el comienzo de la experiencia se detectaron muchos problemas sobre los que será preciso reflexionar.

Existen errores de gestión del recurso desde la perspectiva del docente (haber realizado un perfil en lugar de una página) y también derivados de la propia estructura de las redes sociales. En España estos recursos se asocian con actividades lúdicas y no formativas, si bien la solución podría estar en la conjunción de ambas perspectivas.

Las facilidades para la puesta en marcha de un espacio para una materia docente en las redes sociales se transforman en complicaciones cuando se trata de determinar los contenidos más adecuados para ese espacio. Hoy por hoy, la utilización de este recurso garantiza la recepción de los contenidos, pero no el procesamiento del mismo $\mathrm{y}$, por lo que se ha comprobado, tampoco aporta mejoras en la discusión y en el debate.

El hecho de haber puesto en marcha esta experiencia en el Grado en Publicidad y Relaciones Públicas incide en la importancia de este recurso de una forma más profunda, ya que los estudiantes tendrán que familiarizarse con esta herramienta desde una perspectiva no sólo lúdica y ni tan siquiera académica, sino profesional.

\section{BIBLIOGRAFÍA}

AIMC (2011) "13 $13^{\mathrm{a}}$ Encuesta Navegantes en la Red" en Asociación Española de Investigación en Medios de Comunicación (AIMC). Febrero, 2011. Disponible en http://download.aimc.es/aimc/navred2010/macro2010.pdf Consultado el 22 de agosto de 2011.

Bautista, Guillermo; Borges, Federico y Forés, Anna (2004) Didáctiva universitaria en entornos virtuales de enseñanza-aprendizaje. Madrid. Narcea.

Cabero, Julio (dir.) (2003) “Las nuevas tecnologías en la actividad universitaria” Pixel 
Bit: Revista de medios y educación. $\mathrm{N}^{\mathrm{o}}$ 20. 81-100. Disponible en: http://www.sav.us.es/pixelbit/pixelbit/articulos/n20art/art2008.htm Consultado el 18 de agosto 2011

Caldevilla Domínguez, David (2008): Relaciones Públicas y cultura. Visión Net. Madrid.

Castellón Martínez, Araceli (2010) "Una nueva figura profesional: el community manager" en Pangea: Revista de la Red Académica Iberoamericana de Comunicación. No1. Disponible on line en http://dialnet.unirioja.es/servlet/articulo?codigo=305401 Consultada el 22 de agosto de 2011.

Corbacho Valencia, Juan Manuel (2010) “Redes sociales y normas de urbanidad" en VV.AA. (2010) Nuevos medios, nueva comunicación. Salamanca. Universidad de Salamanca.

De Pablos Pons, Juan y Villanueva Moreno, Patricia (2005) “El Espacio Europeo de Educación Superior y las Tecnologías de la Información y la Comunicación. Percepciones $\mathrm{u}$ demandas del profesorado" en Revista de Educación (convergencia europea y universidad). No 337. Mayo-agosto. 99-124.

IAB (2010) “Informe Redes Sociales en España, 2010” en IAB. Diciembre de 2010. Disponible on line en

http://www.iabspain.net/ver.php?mod=descargas\&id_categoria=4,14,40,17

Consultado el 22 de agosto de 2011.

IAB y Elogia Ipsofacto (2009) “Estudio sobre las redes sociales en internet" en IAB. 10 de noviembre de 2009.

http:/ / www.iabspain.net/ver.php?mod=descargas\&id_categoria=4,14,40,17

Consultado en 22 de agosto de 2011.

Imaña Serrano, Tanya (2008) "Facebook: tejiendo las telarañas de las redes sociales" en Razón y Palabra, nº 2. En http://www.razonypalabra.org.mx Consultado el 22 de agosto de 2011

Infoadez (2010) “Inversión publicitaria en España, 2010" Diciembre de 2010. Disponible on line en http:// www.infoadex.es/RESUMEN\%202010.pdf Consultado el 22 de agosto de 2011.

Inteco (2011) “Estudio sobre seguridad de las comunicaciones móviles e inalámbricas en hogares españoles (3er Trimestre, 2010)" en Instituto Nacional de Tecnologías de la Comunicación (INTECO) e Asociación Española de Protección de Datos (AEPD). Febrero de 2011. Disponible on line en

http://www.inteco.es/Seguridad/Observatorio/Estudios_e_Informes/Estudios_e_In formes_1/estudio_inalambricas_3T2010 Consultado el 22 de agosto de 2011.

Jiménez Garro, Raquel (2011) “El papel de las redes sociales en la eduación” en 
Educaweb.com. En http://www.educaweb.com/noticia/2011/01/31/papel-redessociales-educacion-14577.html Consultado el 27 de febrero de 2011

Marín Díaz, Verónica y Maldonado Berea, Guadalupe Aurora (2010) “El alumnado universitario cordobés y la plataforma virtual Moodle" en Pixel Bit: Revista de medios y educación. $\quad \mathrm{N}^{\mathrm{o}}$ 38. 121-128. En: http://www.sav.us.es/pixelbit/actual/9.pdf Consultado el 18 de agosto de 2011

Meso Ayerdi, Koldo; Pérez Dasilva, Ángel y Mendiguren, Teresa (2011) “Estrategias de enseñanza formal e informal. La incorporación de las redes sociales como herramienta en el aula" en SIERRA SÁNCHEZ, Javier y SOTELO GONZÁLEZ, Joaquín (coords.)(2011) Métodos de innovación docente aplicados a los estudios de Ciencias de la Comunicación. Editorial Fragua. Madrid. 461-473.

Pisticelli, A., Adaime, J.; Binde, I. (2010) El proyecto Facebook y la posuniversidad. Fundación Telefónica. Madrid.

Pozo, Juan Ignacio et al. (2006) Nuevas formas de pensar la enseñanza y el aprendizaje. Las concepciones de profesores y alumnos. Barcelona. Graó.

Salinas, Jesús (2004) "Innovación docente y uso de las TIC en la enseñanza universitaria" en Revista Universidad y Sociedad de Conocimiento. Vol. 1, nº 1 , noviembre de 2004.

Serraller, Mercedes (2009) "El derecho a la intimidad se redefine en internet" en Expansion.com. En: $\quad$ http://www.expansion.com/2009/04/12/juridico.html Consultado el 27 de febrero de 2011

Valverde Berrocoso, Jesús y Garrido Arroyo, María del Carmen (1999) “El impacto de las tecnologías de la información y la comunicación en los roles docentes universitarios" en Revista Electrónica Interuniversitaria de Formación Profesorado. № 2 (1).

\section{Emma Torres Romay}

Profesora contratada doctora de la Facultad de Ciencias Sociales y de la Comunicación donde imparte la materia de Estrategias de la Comunicación Publicitaria. Ha desarrollado su labor investigadora en los campos de la estrategia publicitaria y de la comunicación institucional publicando numerosos artículos sobre estas temáticas en publicaciones de reconocido prestigio. En el campo de la gestión, ocupó el cargo de secretaria del centro durante tres años siendo, en la actual, la coordinadora del Grado en Publicidad y Relaciones Públicas.

\section{Juan Manuel Corbacho Valencia}

Profesor de Relaciones Públicas y Protocolo en el Grado de Publicidad y Relaciones Públicas (Facultad de Ciencias Sociales y de la Comunicación - Universidad de Vigo) 
desde el 2004. Licenciado en Publicidad y Relaciones Públicas y Traducción e Interpretación, así como Doctor en Comunicación, Publicidad y Relaciones Públicas, versando su tesis sobre la publicidad internacional. Autor de numerosas contribuciones a congresos nacionales e internacionales en la actualidad es Decano del centro. 